Editorial

\title{
Professional burnout in anaesthesia and critical care - how to decrease it
}

Volume 2 Issue 3 - 2015

\section{Editorial}

Professional burnout is an entity seen in professionals and is characterized by emotional exhaustion, depersonalization and ineffectiveness. Emotional exhaustion is considered the initial sign of burn out and is primarily caused by mismatch in demands and personal capability to meet them. Depersonalization is characterized by emotional instability and ineffectiveness suggests a negative selfassessment due unhappiness and dissatisfaction at work. ${ }^{1,2}$ Clinical symptoms are nonspecific and include tiredness, headaches, eating problems, insomnia, irritability, emotional instability and rigidity in relationships with other people., This commonly occurs due to imbalance between the demands being made and the ability to meet those demands. Anaesthesia and critical care specialties involve a around-the-clock care to a variety of patients, especially critical patients, in giving perioperative care which requires collection and evaluation of data, development and implementation of strategies to maintain the desired safe patient status. These demands make the humans responsible to provide safe operations, stretch and strain their physiological system to the limits. ${ }^{5}$

A recent French investigation revealed existence of stress in as high as $29.8 \%$ of professionals working in the operating theatre. ${ }^{6}$ Also, in another survey, majority of Irish residents in anaesthesiology felt that work-related stress affected their health and performance. ${ }^{1}$ The human physiology follows a circadian pattern of alertness and performance and needs vital time for sleep and breaks to avoid burn out. Unregulated duty hours and demanding job often leads to development of burnout in anesthesiologists. This can impair the individual's cognitive function. Poor vigilance due to lack of rest and sleep can lead to errors and thereby increase morbidity/mortality. Now-a-days there is a stress on quality and patient safety.

\section{Common causes of burnout}

Various causes have been found related to professional burnout in anaesthesia and critical care professionals. These include:

Sleep deficit: The appropriate number of sleep hours is essential to maintain physiological homeostasis. Inadequate sleep may lead to day time sleepiness and impaired performance. The anaesthesiologist have shift duties and irregular work hours (due to type of surgical cases specially in tertiary care centres with complex cases) that lead to irregular sleep patterns. ${ }^{7}$ This sleep loss gets accumulated and may reduce the ability of individual to perform sustained work. Inadequate sleep has been known to increase accidents. It has been well proven that inadequate sleep or rest can lead to fatigue which in turn can cause decrease attention, prolonged reaction time, impair memory and decision making. ${ }^{3,4,7,8}$ This can compromise safe anaesthesia practice and can lead to errors, critical incidents and accidents if not dealt with properly.

Fatigue and stress: A stressed person gets fatigued earlier than a relaxed person. There are some inherent stressors for a particular profession but unnecessary stressors must be removed to improve performance and reduce fatigue for the anesthetist. Performance of

\author{
Nishkarsh Gupta,Anju Gupta, Rakesh Garg \\ Department of Anaesthesiology, All India Institute of Medical \\ Sciences, India
}

Correspondence: Nishkarsh Gupta, Department of Anaesthesiology, Pain and Palliative Care, Dr BRAIRCH, All India Institute of Medical Sciences, Room No. 139, Ist Floor, Ansari Nagar, New Delhi-I I0029, India, Tel I23 I23 I23, Email drnishkarsh@rediffmail.com

Received: April 13, 2015 | Published: May 12, 2015

Anesthetist can be improved by reducing physical stress (confined space, extreme of temperature), psychological stress (relationship with surgical colleagues, fear of complications and death of patient), mental stress (difficult patients and complex surgical interventions) and physiological stress (unwell resulting in the body using more energy fighting the illness and hence less energy to perform vital tasks, not having proper meals also result in not having enough energy and induces symptoms like headache and shaking, lack of sleep). ${ }^{9}$ Fatigued anesthetist is unable to maintain performance standards for long periods as he is struggling to stay awake due to long working hours. Conflicting shift schedules and working long hours without break affect the body's circadian cycle and lead to a degradation of performance..$^{10-12}$ In various studies fatigue was found to be an important cause of error in medical judgment. ${ }^{13}$ A study reported an increased incidence of unintentional Dural puncture during epidural anaesthesia at night (midnight to 6:00 AM) ${ }^{14}$ In the report of critical incidents to the Australian Incident Monitoring Study from 1987 to 1997 revealed that fatigue was listed as a contributing factor in 152 reports $(3 \%) .{ }^{15}$ Most of us also have also experienced that there is an increased risk of critical incidents between $2 \mathrm{AM}$ to $5 \mathrm{AM}$ in the morning.

Economic and workforce limitations: Aviation industry is continuously making efforts to reduce fatigue and stress among their pilots and thereby reducing the error. But ironically the standards for anesthesiologist are far behind that of aviation industry. Unfortunately we tend to cover up error and deny fatigue and stress. So, we are not able to get an opportunity to improve the working conditions.

Lack of accomplishment: The anesthesiologists don't get acknowledged despite all the efforts made for safe anesthesia. ${ }^{1}$. The patients also hardly know about anesthesiologists and process of anesthesia. ${ }^{16}$ Also many anesthesiologists tend feel isolated because they have only brief contact with the patients, residents and other anesthesiologists throughout the day. The excessive working hours, job dissatisfaction and fatigue may lead to syndrome of emotional exhaustion, depersonalization, and reduced personal accomplishment. This burn out syndrome may lead to problems like alcoholism, 
substance abuse, marital problems, emotional disorders, decreased empathy and psychological withdrawal from work. ${ }^{1-3}$ So, measures need to be taken to improve the work environment. This leads to depression and other psychiatry illness and are prone to drug addiction. The incidence of abuse is higher than general physicians and estimated to be around $1 \%$ in faculty members and $1.6 \%$ of residents. ${ }^{17}$

The commonly abused drugs include opioids(morphine, fentanyl and sufentanil), propofol, ketamine, nitrous oxide and volatile anesthetics. ${ }^{18-21}$ Addiction to drugs impairs the health care professional and makes them vulnerable. This can put the life of the patients in danger and increase the professional liability for himself and his group in case of lawsuit. It may be difficult to identify an impaired anesthesiologist as they tend to self-diagnose and treat themselves. The warning signs include absenteeism from work, changes in sleeping and eating patterns, mood swings, arriving late and conflicts with colleagues should raise the suspicion of a possible substance abuse.

Impact of burn out: Traditionally process of giving anaesthesia is compared with flying an aeroplane. An airline pilot is only permitted to fly 8 hours in a $24 \mathrm{hr}$ period, 30 hours in 7 days, 100 in a month and up to 1,000 hours in a calendar year by FAA regulation. Anesthesiologists in the interest of patients, sometimes have to provide their services for extended hours. Usually, duty hours generally depend on the number of anesthetist available, which sometimes may make extended working hours for anaesthesiologists. Sleep deprivation can also increase gastrointestinal and cardiovascular diseases, increases adverse pregnancy outcomes, increases risk of breast cancer, decreases immunity, impairs glucose metabolism and leads to a decrease in cerebral metabolic rate and psychological diseases. ${ }^{2,3,8}$

Pre-operative/Preflight stress of a pilot is similar but more problematic for anesthetist as a pilot will not fly the plane unless ground staff or maintenance staff gives $100 \%$ fitness to airplane, but anesthetist has to depend on physicians for pre-operative preparations but with no responsibility and patient has to be taken up invariably with calculated risk. Take-off is similar to induction of anaesthesia, which requires multitasking, skills, vigilance, dependence on assistance and equipment. It involves physical, psychological, mental alertness, full concentration and margin of safety is very narrow. Maintenance phase is similar to flight with turbulences which may or may not be anticipated. Level of alertness, vigilance and concentration cannot be lowered and duration of this period cannot be anticipated most of the time. Landing is again similar to reversal of anaesthesia which is the most difficult time in the whole process, requiring mental capability and skill more than at the time of induction and margin of safety is at the lowest in this phase as most of the controls are being released slowly. Taxing of plane is similar to post-operative period but stress on anesthetist does not end as he is accountable if any problem occurs but vigilance of this period is under surgeon and post-operative staff, meaning responsibility with minimum control.

Minimum monitoring standards mention a qualified anesthetist as a first priority. But nothing has been mentioned to standardize work hours for anesthetist. It has been well proven that the handeye coordination may be impaired after long duty hours (impairment may be similar to a blood alcohol level of $0.05 \%-0.1 \%)$. $^{11,12}$ This can increase the risk of injuries to the doctor and could have deleterious effects on patient safety.

\section{Measures to prevent burnout in anesthesiologist}

It is complex situation and one has to consider the nature of job (24/7/365days), differences among anesthetists (age, sex, experience etc.), and increasing complexities of the patients. It has been seen that an older anesthesiologist better tolerates the stress than a novice. ${ }^{2}$

A comprehensive approach is required to improve the overall situation. It should include:

a. In medical school we are taught complexities of various diseases, but we are not taught basics in sleep medicine, sleep disorders and to work in optimum conditions. If we don't educate ourselves we cannot move towards a change.

b. Efforts should be taken to introduce various strategies to improve alertness in our curriculum

c. There should be a provision of planned naps after a stipulated duration of work. Various studies have shown that planned naps can improve subsequent alertness and performance. A NASA field study suggested a 40-min nap increased performance (34\%) and physiologic alertness (54\%) compared with a nonap condition. We should have discussion on it and come to a reasonable conclusion.

d. It is well known that caffeine ingestion is known to be a stimulant to maintain wakefulness. Its onset occurs about 15-30 min after ingestion and its effect lasts about 3-4h. its usefulness is limited because of adverse reactions like tremors and palpitations.

e. One should take into consideration maximum duty hours, minimum rest opportunities, side effects of inadequate sleep, start and end times of shifts and cumulative fatigue and make scheduling policies. We should have strict regulations to limit the number of work hours per week/month. The maximum duty at a stretch should not be more than 12 hours.

f. One should have a compulsory one week paid leave for a holiday every six months irrespective of the stage of career.

g. Lounges with facilities for recreation (newspapers, treadmill etc.) should be located in close vicinity to work place so that we can de-stress ourselves.

h. Anesthetist should compulsorily be relieved for 30minutes every 4- hours for de-stressing themselves and technique of de stressing should be taught on regular intervals.

i. Stress management workshops should be conducted at regular intervals to help manage stress full lives.

j. There should be provision for refreshments/food for anesthetist working beyond routine hours.

k. There should be designated comfortable separate rooms for both genders adjacent to the operating room for night duty anesthetist for sleep and rest during post call.

1. Only lifesaving, limb saving surgery to be performed during night hours.

$\mathrm{m}$. There should be reduced duty hours for seniors and their experience should be utilized in improving teaching and research.

n. Ratio of Anesthetists vs. Number of operating rooms in a hospital and ratio of Anesthetists Vs number of surgeons in a hospital must also be calculated and defined for optimum utilization of Operating rooms and surgeons.

o. Time for study, case discussions, auditing, research, attending CME and workshops should also be included in working hours of the anesthetists. 


\section{Conclusion}

Burnout syndrome may be considered a marker of the health of the caregiver team. It may be seen and anesthesiologist and has an important impact on daily quality of life and may threaten patient care. Moreover, several determinants of severe burnout are related to organization, duration of working hours, conflicts, communication among healthcare workers, and the management of end-of-life care. Efforts should be made to recognize burnout in anesthesiologist and intensivists and suggest solutions to the problem.

\section{Acknowledgements}

None.

\section{Conflicts of interest}

The authors declare that there are no conflicts of interest.

\section{Funding}

None.

\section{References}

1. Walsh AM, Mc Carthy D, Ghori K. Anesthesiology resident burnout-an Irish perspective. Anesth Analg. 2014;118(2):482-483.

2. Shams T, El-Masry R. Job Stress and Burnout among Academic Career Anaesthesiologists at an Egyptian University Hospital. Sultan Qaboos Univ Med J. 2013;13(2):287-295.

3. Mion G, Libert N, Journois D. Burnout-associated factors in anaesthesia and intensive care medicine. 2009 survey of the French Society of anesthesiology and intensive care. Ann Fr Anesth Reanim. 2013;32(3):175-188.

4. Grau A, Suner R, Garcia MM. Burnout syndrome in health workers and relationship with personal and environmental factors. Gac Sanit. 2005;19(6):463-470.

5. Kohn LT, Corrigan JM, Donaldson MS. To Err Is Human: Building a Safer Health System. Washington DC, USA: National Academy Press; 1999. pp. 312.

6. Travers V, Watrelot A, Cuche H. Evaluation of the level of stress and indicators in physicians working in the operating room [in French]. Presse Med. 2012;41(12 Pt 1):e577-585.

7. Wisetborisut A, Angkurawaranon C, Jiraporncharoen W, et al. Shift work and burnout among healthcare workers. Occup Med (Lond). 2014;64(4):279-286.
8. Cassidy CJ, Griffiths E, Smith AF. Safety in sleep: anaesthetists, patients and the European Working Time Directive. Anaesthesia 2014;59(9):841-843.

9. Ayas NT, Barger LK, Cade BE, , et al. Extended Work Duration and the Risk of Self-reported Percutaneous Injuries in Interns. JAMA. 2006;296(9):1055-1062.

10. Barger LK, Ayas NT, Cade BE, et al. Impact of extended-duration shifts on medical errors, adverse events, and attentional failures. PLoS Med. 2006;3(12):e487.

11. Embriaco N, Azoulay E, Barrau K, et al. High level of burnout in intensivists: prevalence and associated factors. Am J Respir Crit Care Med. 2007;175(7):686-692.

12. Williamson AM, Feyer AM. Moderate sleep deprivation produces impairments in cognitive and motor performance equivalent to legally prescribed levels of alcohol intoxication. Occup Environ Med. 2009;57(10):649-655.

13. Montgomery A, Todorova I, Baban A, et al. Improving quality and safety in the hospital: the link between organizational culture, burnout, and quality of care. Br J Health Psychol. 2013;18(3):656-662.

14. Okell RW, Sprigge JS. Unintentional dural puncture:A survey of recognition and management. Anaesthesia. 1987;42(10):1110-1113.

15. Morris GP, Morris RW. Anaesthesia and fatigue:an analysis of the first 10 years of the Australian Incident Monitoring Study 1987-1997. Anaesth Intensive Care. 2000;28(3):300-304.

16. Kluger MT, Townend K, Laidlaw T. Job satisfaction, stress and burnout in Australian specialist anaesthetists. Anaesthesia. 2003;58(4):339-345.

17. Booth JV, Grossman D, Moore J, et al. Substance abuse among physicians:a survey of academic anaesthesiology programs. Anesth Analg. 2002;95(4):1024-1030

18. Wischmeyer PE, Johnson BR, Wilson JE, et al. A Survey of Propofol Abuse in Academic Anesthesia Programs. Anesth Analg. 2007;105(4):1066-1071.

19. Fry RA. Substance abuse by anaesthetists in Australia and New Zealand. Anaesth Intensive Care. 2005;33(2):248-255

20. Garland EL, Howard MO, Perron BE. Nitrous oxide inhalation among adolescents:prevalence, correlates, and co-occurrence with volatile solvent inhalation. J Psychoactive Drugs. 2009;41(4):337-347.

21. Wilson JE, Kiselanova N, Stevens Q, et al. A survey of inhalational anaesthetic abuse in anaesthesia training programmes. Anaesthesia. 2008;63(6):616-620. 